\title{
Radiomics - Using Artificial Intelligence in the Quest towards Personalised Radiation Treatment
}

\author{
Ahmed Nadeem Abbasi, Agha Muhammad Hammad Khan and Bilal Mazhar Qureshi
}

The term radiomics is a newer concept evolved over the past two decades. Imaging data is stored in a data-base that can be utilised in order to process and review variations in treatment outcomes. ${ }^{1}$ Artificial intelligence (Al) and Big Data is evolving with practical implementation in a number of areas in the healthcare systems resulting in improvement of patient care and medical related outcomes. ${ }^{2}$ The future trend is steering towards dependency on encrypted digitalised data storage. Out of these data, one is related to diagnostic radiology which includes information in the form of radiographs, ultrasound scans, mammograms, CT scans, MRIs, and metabolic imaging such as PET/CT scan that may have a potential to address multiple oncology related queries by improving diagnostic, prognostic, and predictive accuracy of cancer management. Treatment related response can be validated in such a way that it can be further adapted with the concept of 'personalised' treatment in radiation oncology. ${ }^{3}$

Imaging, being a non-invasive procedure, is being used during multiple phases of a patient's treatment journey. Imaging is done for diagnosis, staging, radiation planning, and response assessments; giving us an edge of tracking the tumor from the beginning till end. It allows a clinician to visualise a tumor through all these phases. Treatment has evolved from gray scales algorithms to fusion of images and then to functional imaging, leading to modification of treatment plans. ${ }^{4}$

Summation of existing data is a real challenge due to heterogeneity in protocol used at different institutions. Limitations arise when we review the literature, which show that there is no standardisation of definition of imaging data. Most of the studies used their in-house developed software, which was not shared in masses, making it an exclusive thing that is challenging its potential of changing the dynamics of future of oncology treatment. A number of variations do exist while

Department of Oncology, The Aga Khan University Hospital, Karachi, Pakistan

Correspondence: Dr. Ahmed Nadeem Abbasi, Department of Oncology, The Aga Khan University, Stadium Road, Karachi, Pakistan

E-mail: nadeem.abbasi@aku.edu

Received: October 10, 2018; Accepted: November 19, 2018 acquiring data, which includes noise corrections, difference in vendor setting for every equipment, dynamic changes in the form of progression, and shrinkage of tumors and organ motion. These variations can be managed by using appropriate maneuvers. A four-step model has taken into account for processing all the radiological data. Lambin et al. described this flow and identified the steps. These include imaging, segmentation, feature extraction, and analysis. First step is identifying imaging data to start the process followed by software-based automated segmentation of structures to avoid intra- and inter-subjective variability, leading to feature extraction. Taking an example of CT imaging, the most common imaging modality used in oncology practice: identified intensity, textures, wavelet and shape features can be used to evaluate temporal relation with genetics and survival models. The final step is analysis by a radiomic software that will predict model and validation by making use of statistic models. ${ }^{5}$

Many studies have been conducted in order to identify radiological association with genomic, cellular, molecular, metabolic, and clinical data. More the data is combined, greater are the chances of identifying statistic proven relation with radiological data. 6 Stephen et al. reported genotype-phenotype interaction between FDGPET and EGFR mutations, enlightening the use of noninvasive imaging as a tool to predict a mutation in nonsmall cell lung cancer. ${ }^{7}$ A study conducted on a cohort of 110 patients with grade II glioma tumors showed association of IDH1 mutation with T2-Flair images with area under the ROC (receiver-operating characteristic) curve to 0.86 .8 Similar results were concluded from a glioblastoma-based study, where radiological data was extracted with 12-month survival rate and EGFR mutation. ${ }^{9}$ Parmer et al. identified multiple radiomic clusters with patient survival and staging in head and neck and lung cancers with significant $p$-value (Lung $R S=0.92$, $p<0.001, H \& N$ RS $=0.92, p<0.001)$. Moreover, on multivariate analysis radiomic features were also related to predictive models. ${ }^{10}$ Radiomic features can be correlated with gene expression, mutation, histopathological grading, hormone status, micro-vascular density, tumor metabolism, and malignancy predicltion.

At this moment, it is very difficult for a radiation oncologist to confess that radiomic data, on its own, can influence and/or alter patient's radiation treatment. As discussed previously, it is imperative to utilise other data sets as 
well, including clinical, genomics, and proteomics. Aerts et al. computed that if all the data are merged and processed, its power of predicting the development of an association can be enhanced; and it can give more power to a study. The greater the cohorts for comparisons with radiological data, the better the chances for implementation and further evolution for personalised treatment in the area of oncology. 6

The concept of the recently proposed virtual biopsy will challenge the current model of oncologic management; which has a pathway of imaging, followed by surgical resection. A single-point tissue biopsy may decide the whole treatment process, ignoring the tissue heterogeneity that may not only exist within the tumor specimen but also missing heterogeneity that may exist in other distant parts of the body. With the help of radiomics, these virtual biopsies will have a pathway of radiomic assessment without tissue extraction. Multiple sites can be evaluated with a non-invasive technique, giving chance to determine and alter treatment which is not based on single-tissue specimen. Hence, the heterogeneity that current model cannot pick up due to its practical limitations. ${ }^{11}$

The whole concept spins around developing and consolidating personalised oncology practice, where each and every patient should be treated as per his or her need to prolong overall survival and minimising toxicities. Hence, improving therapeutic ratio. This notion explains that no two individuals are alike, which will challenge the current concept of making cohorts and treating them on same pattern because outcomes do vary due to heterogeneity in tumors because of genetic configuration. Developments of CDSS (clinical decision support system) will analyse radiological data to help healthcare providers make decisions and improve patient care. ${ }^{12}$ Many radiation oncologists believe that personalised radiation treatment is the future of radiation oncology; and radiomics will become the new face of radiation therapy in the upcoming years. The jury is still out, we must confess that the data is emerging; and at this moment, it is not fully mature. Advent of concepts of radiomics offers a potential opportunity towards positive changes in the understanding of radiation oncology.

\section{REFERENCES}

1. Bourgier $C$, Colinge J, Aillères $N$, Fenoglietto $P$, Brengues $M$, Pèlegrin $A$, et al. Radiomics: Definition and clinical development. Cancer Radiother 2015; 19:532-7.

2. Luo J, Wu M, Gopukumar D, Zhao Y. Big data application in biomedical research and healthcare: A literature review. Biomed Inform Insights 2016; 8:1-10

3. Gillies RJ, Kinahan PE, Hricak H. Radiomics: images are more than pictures, they are data. Radiology 2015; 278:563-77.

4. Pereira GC, Traughber M, Muzic RF Jr. The role of imaging in radiation therapy planning: past, present, and future. Biomed Res Int 2014; 2014:231090

5. Lambin P, Leijenaar RTH, Deist TM, Peerlings J, de Jong EEC, van Timmeren J, et al. Radiomics: the bridge between medical imaging and personalized medicine. Nat Rev Clin Oncol 2017; 14:749-62.

6. Aerts HJ, Velazquez ER, Leijenaar RT, Parmar C, Grossmann P, Carvalho $\mathrm{S}$, et al. Decoding tumour phenotype by noninvasive imaging using a quantitative radiomics approach. Nat Commun 2014; 5:4006.

7. Yip SS, Kim J, Coroller TP, Parmar C, Velazquez ER, Huynh E, et al. Associations between somatic mutations and metabolic imaging phenotypes in non-small cell lung cancer. J Nucl Med 2017; 58:569-76.

8. Yu J, Shi Z, Lian Y, Li Z, Liu T, Gao Y. Noninvasive IDH1 mutation estimation based on a quantitative radiomics approach for grade II glioma. Eur Radiol 2017; 27:3509-22.

9. Lee J, Narang S, Martinez JJ, Rao G, Rao A. Associating spatial diversity features of radiologically defined tumor habitats with epidermal growth factor receptor driver status and 12-month survival in glioblastoma: methods and preliminary investigation. J Med Imaging (Bellingham) 2015; 2:041006.

10. Parmar C, Leijenaar RT, Grossmann P, Velazquez ER, Bussink J, Rietveld $\mathrm{D}$, et al. Radiomic feature clusters and prognostic signatures specific for lung and head \& neck cancer. Sci Rep 2015; 5:11044.

11. Sanduleanu S, Woodruff HC, de Jong EE, van Timmeren JE, Jochems A, Dubois $L$, et al. Tracking tumor biology with radiomics: A systematic review utilizing a radiomics quality score. Radiother Oncol 2018; 127:349-60.

12. Lambin P, Zindler J, Vanneste BG, Van De Voorde L, Eekers D, Compter I, et al. Decision support systems for personalized and participative radiation oncology. Adv Drug Deliv Rev 2017; 109:131-53.

$$
\text { …두.... }
$$

